\title{
Screening for TB by sputum culture in high-risk groups in Copenhagen, Denmark: a novel and promising approach
}

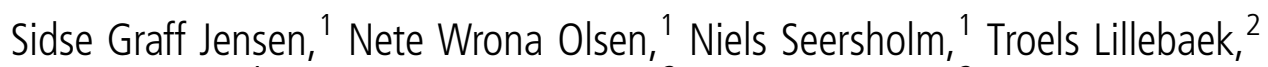 \\ Torgny Wilcke, ${ }^{1}$ Mathias Klok Pedersen, ${ }^{2}$ Axel Kok-Jensen ${ }^{2}$
}

${ }^{1}$ Department of Respiratory Medicine, Gentofte Hospital, Hellerup, Copenhagen, Denmark

${ }^{2}$ International Reference Laboratory of

Mycobacteriology, Statens Serum Institut, Copenhagen, Denmark

\section{Correspondence to} Dr Sidse Graff Jensen, Department of Respiratory Medicine, Gentofte Hospital, Kildegaardsvej 28, Hellerup, Copenhagen 2900, Denmark; Sidse.graff.jensen@regionh.dk

Received 10 April 2015 Accepted 18 June 2015 Published Online First 8 July 2015
CrossMark

\footnotetext{
To cite: Jensen $\mathrm{SG}$, Olsen NW, Seersholm N, et al. Thorax 2015;70: 979-983.
}

\begin{abstract}
Introduction Evidence on screening high-risk groups for TB by mobile X-ray in low-incidence countries is building, but knowledge on other possible screening methods is limited. In this retrospective study we report results from a community based programme screening for TB by spot sputum culture.

Methods On seven occasions, from September 2012 through June 2014, we offered TB screening to all persons present at 11 locations where socially

marginalised people gather in Copenhagen. Spot sputum samples from participants were examined by smear microscopy and culture. Genotype, nucleic acid amplification test and chest X-ray were done if TB was found.

Results Among 1075 participants, we identified 36 cases of TB. Twenty-four cases (66.7\%) were identified at the first screening of each participant, that is, the prevalence of TB was 2233/100 000. Thirty-five (97\%) of the TB cases were culture-positive and seven (19.4\%) were smear-positive. Twelve out of $21(57.1 \%)$ cases tested were nucleic acid amplification test positive. Twenty-eight (77.8\%) had chest X-ray suggestive of TB. All patients with TB started treatment, 30 (83.3\%) had a successful outcome.
\end{abstract}

Discussion Screening for TB by spot sputum culture is possible and a promising alternative to mobile $\mathrm{X}$-ray in a community based screening programme. $22.2 \%$ did not have chest $X$-ray suggestive of TB and would not have been identified using mobile $X$-ray. Most of the TB cases were smear-negative, suggesting that they were identified at an early, less infectious stage, which is essential in order to prevent transmission and gain infection control.

\section{INTRODUCTION}

In low-incidence countries, TB is concentrated in high-risk groups including socially marginalised persons (eg, homeless persons and alcoholics). ${ }^{1} 2$ These groups are hard to reach in terms of diagnosis. Therefore, delayed diagnosis and ongoing transmission is frequent. ${ }^{3} 4$ In order to facilitate early diagnosis, active case finding is recommended and screening of high-risk groups is one of the priority areas defined by WHO in the recently published 'Framework towards TB elimination in lowincidence countries'.

Several studies on mobile X-ray screening of homeless persons have been published. ${ }^{6-10}$ Data on

\section{Key messages}

What is the key question?

- Is it feasible to screen high-risk groups for TB using spot sputum culture in a TB low-incidence country?

\section{What is the bottom line?}

- We provide evidence that screening by spot sputum culture is possible and a promising alternative to mobile $\mathrm{X}$-ray screening; furthermore TB cases identified in our study were found in an early, less infectious stage of their disease, which is important in order to gain disease control in high-risk groups.

\section{Why read on?}

- Screening for TB in high-risk groups in low-incidence countries is recommended by WHO; this is the first study on systematic, repeated, community-based screening for TB by spot sputum culture in a larger high-risk group $(n=1075)$ in a TB low-incidence country.

other possible screening tools such as symptom screening, sputum examination and nucleic acid amplification test (NAAT) (eg, the Xpert MTB/RIF assay) are limited. ${ }^{3}$ To our knowledge, only two studies on screening homeless persons by sputum culture have been published including 95 and 120 participants, respectively. ${ }^{11} 12$

In 2012, the TB incidence rate was $6.9 / 100000$ in Denmark and 17.0/100000 in Copenhagen (1.25 million inhabitants). ${ }^{13}$ TB is a prevalent problem among socially marginalised persons (ie, homeless persons, persons with alcohol abuse and drug abuse) in Copenhagen, among whom high rates of transmission of Mycobacterium tuberculosis have been reported. In particular one predominant strain of M. tuberculosis (Cluster 2/1112-15) has increased indicating active transmission. ${ }^{14-16}$ Another TB high-risk group in Denmark is Greenlandic Inuit. Greenland (approximately 56500 inhabitants) is a part of the Kingdom of Denmark and many Greenlandic Inuit live in Denmark. Although most do well in the Danish society, $11 \%$ of homeless people in Copenhagen in 2013 were Greenlandic Inuit. ${ }^{17}$ TB incidence rate 
in Greenland 2006-2010 was 132/100 000; in Denmark 29\% of all patients with TB in 2012 were Greenlandic Inuit (estimated incidence 500/100 000). ${ }^{13}$

In the Copenhagen area, the conventional mobile X-ray screening for TB was not available after 2011. Instead spot sputum screening among socially marginalised persons, including socially marginalised Greenlandic Inuit, was implemented. Sputum samples were collected for smear microscopy, culture, and in some cases NAAT.

In this retrospective study we report results from this community based programme. We assess whether it was possible to identify TB cases by spot sputum culture and to find the participants again and initiate TB treatment when the culture proved positive. Furthermore we compare results from different diagnostic methods (chest X-ray at time of diagnosis, smear microscopy, sputum culture and NAAT) when available.

No previous studies of systematic, repeated, community based screening for TB using spot sputum culture in high-risk groups in a TB low-incidence country have been published.

\section{METHODS}

\section{Screening procedure}

From September 2012 through June 2014, TB screening by sputum culture was offered to all persons present at 11 locations in Copenhagen. These were locations where homeless persons; persons with alcohol and/or substance abuse; and other socially marginalised persons, including socially marginalised Greenlandic Inuit, gather. It was locations in which TB cases had been identified previously and included shelters, soup kitchens, selected public squares and Freetown Christiania (a selfproclaimed autonomous neighbourhood in Copenhagen). TB screening was offered on seven occasions (screening rounds) at each location. The screening sessions were advertised at the locations in advance. Persons present at the screening site were actively contacted and participants were encouraged to be re-examined as many times as possible.

Names, contact information and social security numbers (a personal, unique identification number, which contains date of birth) were registered. If the participant did not have a social security number, date of birth was registered. All sputum samples were collected by a specially trained nurse, who supervised the participants in producing one 'high quality' sputum sample from the lower respiratory tract.

\section{Sample handling and analysis}

All sputum samples were analysed at the International Reference Laboratory of Mycobacteriology, Statens Serum Institut, Copenhagen. The sputum samples were stained for acid-fast bacilli using auramine-rhodamine staining and examined using fluorescence microscopy. Culture was performed in liquid medium (BD BACTEC MGIT 960 System) and on solid medium (Löwenstein-Jensen). Culture-positive isolates were genotyped by the 24-locus based mycobacterial interspersed repetitive unit-variable-number tandem repeat method (genotyping kit: Genoscreen, Lille, France).

Samples from the third of the seven screening rounds and culture-positive samples from the subsequent screening rounds were additionally tested for the presence of M. tuberculosis by a commercially available NAAT, BD ProbeTec (Becton-Dickinson).

The International Reference Laboratory of Mycobacteriology, Statens Serum Institut is accredited in accordance with International Organisation for Standardisation (ISO) 17025 standards and participated in external quality assurance programmes for all diagnostic tests involved in the study with excellent results.

\section{TB cases}

The laboratory promptly informed the screening team of any result suggestive of TB. Participants were then contacted using the contact information given at the time of the sputum collection. Most of them had provided information as to which shelters and/or soup kitchens they would be likely to visit and were contacted there. Others were contacted by telephone, mail or through named contacts.

If sputum samples were culture-positive, smear-positive or NAAT-positive, participants were referred to the Department of Respiratory Medicine, Gentofte Hospital for further examinations. They were identified as patients with TB if their sputum samples were culture-positive or if it was smear-positive or NAAT-positive and the participant had symptoms and/or chest $\mathrm{X}$-ray suggestive of TB.

Data on initiation of treatment, ethnicity, substance abuse, living conditions, comorbidities, previous TB and chest X-ray results as described by or under supervision of an experienced pulmonary physician were obtained from the medical records.

\section{Analysis of data}

Data are presented using median and range.

We calculated the disease prevalence of the screened population as the number of TB cases found the first time the patient was screened divided by the total number of participants screened. To estimate whether repetitive screening was beneficial we divided the number of TB cases identified in the second, third, fourth, etc, screening rounds of the patient with the number of participants who had been screened at least that number of times.

We used McNemar's test to test if there was a significant increase in the number of patients with TB who were smearpositive at initiation of treatment compared with at the time of screening (paired, categorical data). Patients who had not been retested at treatment initiation were not included in this analysis.

When calculating sensitivity and specificity of NAAT, we used sputum culture results as the gold standard.

All statistical analyses were performed using SAS Enterprise guide V.4.3 statistical software (SAS Institute, Cary, North Carolina, USA).

\section{Ethics}

All procedures were done as part of a public health screening programme.

Permission to access and process data was given by the Danish Data Protection Agency (J nr: 2007-58-0015).

\section{RESULTS}

We screened 1075 participants and identified 36 (3.35\%) cases of TB. Figure 1 shows the number of persons screened and number of $\mathrm{TB}$ cases identified in each of the seven screening rounds and table 1 shows their baseline characteristics. Of the screened population, $57.8 \%$ were only screened once, whereas $42.2 \%$ were screened at least twice (table 2).

Of the $36 \mathrm{~TB}$ cases found during the study period, 24 (66.7\% of all TB cases) were identified the first time the participant was screened, that is, the prevalence of TB was $24 / 1075=2.23 \%$ or $2233 / 100000$. Twelve TB cases were initially culture-negative but turned culture-positive in later screening rounds (table 3 ). Time from last negative screening to diagnosis among the patients with $\mathrm{TB}$ had a median of 4 months (range 3-17 months). 


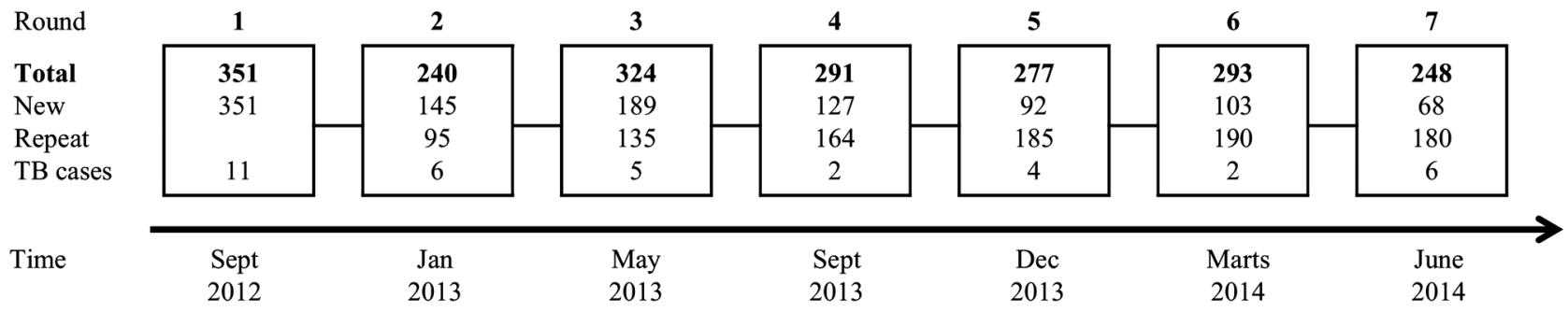

Figure 1 Participants screened in screening round 1-7: total, new, repeat and TB cases identified.

We collected 2024 sputum samples from the participants. Table 4 shows the results of the sputum samples from the $36 \mathrm{~TB}$ cases at the time of $\mathrm{TB}$ diagnosis. In total, 35 (97\%) were culture-positive. One participant was culture-negative, but smear-positive. This patient had symptoms suggestive of TB at the time of the smear microscopy and treatment was initiated. The patient recovered and although repeated cultures were negative, the patient completed treatment and was considered a probable TB case.

The median treatment delay, defined as time from screening to initiation of treatment, was 32 days (range 2-91 days). The median locating delay, defined as time from our receiving screening results from the laboratory until the participant was found, informed and treatment was initiated, was 8 days (range $0-43$ days).

Smear microscopy was done at the time of screening for all patients and for 34 of the 36 patients with TB also at initiation of treatment. At treatment initiation $29.4 \%$ was smear positive versus $19.4 \%$ at screening $(\mathrm{p}=0.34)$.

All 324 sputum samples collected during the third screening round (May 2013) were tested by NAAT. Four were NAAT-positive (two culture-positive and two culture-negative

Table 1 Baseline characteristics of the screened population and the TB cases identified

\begin{tabular}{|c|c|c|}
\hline & $\begin{array}{l}\text { Screened population } \\
\mathrm{n}=1075\end{array}$ & $\begin{array}{l}\text { TB cases identified } \\
\mathrm{n}=36\end{array}$ \\
\hline Age, years* & 45.5 (SD 11.2) & 46.7 (SD 10.0) \\
\hline \multicolumn{3}{|l|}{ Sex } \\
\hline Male & 765 (71.2\%) & $28(77.8 \%)$ \\
\hline Female & $310(28.8 \%)$ & $8(22.2 \%)$ \\
\hline \multicolumn{3}{|l|}{ Ethnicity } \\
\hline Danish & & $6(16.7 \%)$ \\
\hline Greenlandic & & $26(72.2 \%)$ \\
\hline Other-TB high incidence & & $2(5.6 \%)$ \\
\hline Other-TB low incidence & & $2(5.6 \%)$ \\
\hline Homeless & & $23(63.9 \%)$ \\
\hline \multicolumn{3}{|l|}{ Substance abuse } \\
\hline Alcoholt & & $23(63.9 \%)$ \\
\hline Cannabist & & $19(52.8 \%)$ \\
\hline Drugst & & $4(11.1 \%)$ \\
\hline None & & $8(22.2 \%)$ \\
\hline One & & $12(33.3 \%)$ \\
\hline Two or more & & $16(44.4 \%)$ \\
\hline Previous TB & & $6(16.7 \%)$ \\
\hline HIV infection‡ & & $0(0.0 \%)$ \\
\hline
\end{tabular}

with a recent history of TB). Of 329 NAAT-negative samples, three were culture-positive. Using culture results as the gold standard the sensitivity of NAAT was 0.40 and the specificity was 0.99 in this population.

Among the 36 identified TB cases, sputum samples from 21 were tested by NAAT. Twelve (57.1\%) were positive for M. tuberculosis.

Chest X-ray at initiation of treatment was suggestive of TB in $77.8 \%$ of the patients, $22.8 \%$ of patients had a normal chest $\mathrm{X}$-ray. The 35 culture-positive TB cases were genotyped, all with clustered strains. The biggest Danish cluster C2/1112-15 accounted for 24 (68.6\%) of all identified cases.

All 38 participants who had screening tests suggestive of TB were located, contacted and informed; 36 started treatment, 2 were NAAT-positive but had a recent history of TB, no current symptoms suggestive of TB and were culture-negative. Table 5 shows treatment outcome according to WHO definitions on 19 February 2015.

\section{DISCUSSION}

In this study, we provide evidence that spot sputum culture can be used as a screening tool in a systematic, community based screening programme for TB in high-risk groups in a lowincidence country. It is possible to collect sputum samples from relevant participants; to identify TB cases from sputum culture; and to find the participants again and initiate treatment when their sputum culture proves positive. Furthermore, $22.2 \%$ of the TB cases identified did not have chest X-ray suggestive of TB and therefore would not have been identified through mobile $\mathrm{X}$-ray screening.

In settings where sputum culture is available, it remains the gold standard for TB diagnosis, ${ }^{18}$ and is often used as such in diagnostic trials. As a screening tool sputum culture has been dismissed as being too time-consuming and expensive. ${ }^{3}$ It has been used in prevalence studies and to confirm suspicion of TB raised on the basis of other screening methods (symptom

Table 2 Number of participants according to the number of screening rounds they completed

\begin{tabular}{lcc}
\hline Screening rounds completed & \multicolumn{2}{l}{$\begin{array}{l}\text { Participants } \\
\mathbf{n}\end{array}$} \\
\hline One or more & 1075 & Per cent \\
Two or more & 454 & 100 \\
Three or more & 255 & 42.2 \\
Four or more & 144 & 23.7 \\
Five or more & 66 & 13.4 \\
Six or more & 25 & 6.1 \\
Seven & 5 & 2.3 \\
\hline
\end{tabular}


Table 3 Number of TB cases found according to number of screening rounds the participant had completed when TB was diagnosed and as a percentage of the number of participants, who had participated in at least that number of screening rounds

\begin{tabular}{lll}
\hline $\begin{array}{l}\text { Screening rounds } \\
\text { completed at time } \\
\text { of TB diagnosis }\end{array}$ & $\begin{array}{l}\text { TB cases } \\
\mathbf{n}=36\end{array}$ & $\begin{array}{l}\text { Percentage of participants } \\
\text { completing minimum that } \\
\text { number of screening rounds }\end{array}$ \\
\hline 1 & 24 & $2.23 \%$ (=prevalence) \\
2 & 7 & $1.54 \%$ \\
3 & 4 & $1.57 \%$ \\
4 & 0 & $0.0 \%$ \\
5 & 1 & $1.56 \%$ \\
6 & 0 & 0 \\
7 & 0 & 0 \\
\hline
\end{tabular}

screening or chest X-ray). ${ }^{6} 1920$ To our knowledge, only two studies using sputum culture to screen high-risk groups for TB in low-incidence countries have been published. Badiaga et al conducted a prevalence survey of respiratory diseases among homeless people in Marseilles, France on two days in February 2005. Ninety-five of 219 participants provided sputum samples, none of which were culture-positive. In this study, they identified two TB cases by chest X-ray, which indicates that TB prevalence in the study population was $1 \% .^{12}$ In a study from Birmingham, Alabama, Kimerling et $a l^{11}$ screened persons present at two homeless shelters for $\mathrm{TB}$ using symptom and spot sputum screening. The study had two screening rounds 10 months apart, 127 persons were screened; only 9 were encountered twice. Sputum samples were collected from 120 participants, four culture-positive TB cases were identified and TB prevalence was $3.1 \%$. They concluded that spot sputum screening were effective, logistically simple and effective. Our study is larger (1075 participants) and the first study published where systematic, repeated, community based TB screening has been done by spot sputum culture.

Mobile chest X-ray is a good screening tool for TB in highrisk groups as concluded in a recent review. ${ }^{7}$ In this study we found that $8(22.2 \%)$ of the TB cases identified by culture did not have a chest X-ray suggestive of TB at treatment initiation. False positive cultures due to contamination could be an explanation but is not likely. All cultures were performed in a laboratory following ISO 17025 standards, including external quality assurance schemes for all procedures involved. In addition, cultures from all new patients with TB found at the laboratory are routinely genotyped and their DNA patterns compared with patterns from any other culture-positive patients in Denmark

Table 4 Sputum sample and X-ray results from the 36 TB cases identified

\begin{tabular}{lcll}
\hline & Positive & Negative & Not done \\
\hline $\begin{array}{l}\text { Culture } \\
\text { At screening }\end{array}$ & $35(97 \%)$ & $1(3 \%)$ & 0 \\
Smear & & \\
At screening & $7(19.4 \%)$ & $29(80.6 \%)$ & 0 \\
At treatment start & $10(27.8 \%)$ & $24(66.7 \%)$ & $2(5.6 \%)$ \\
NAAT & $12\left(57.1 \%{ }^{*}\right)$ & $9\left(42.9 \%{ }^{*}\right)$ & 15 \\
X-ray & $28(77.8 \%)$ & $8(22.2 \%)$ & 0 \\
\hline *Percentage of 21 tested by NAAT. & & \\
NAAT, nucleic acid amplification test. & &
\end{tabular}

Table 5 Treatment outcome according to WHO definitions

\begin{tabular}{lrl}
\hline Treatment outcome & $\mathbf{n}$ & Per cent \\
\hline Success & 30 & 83.3 \\
Died & 1 & 2.8 \\
Failed & 0 & 0.0 \\
Defaulted & 4 & 11.1 \\
Still on treatment & 1 & 2.8 \\
All & 36 & $100 \%$ \\
\hline
\end{tabular}

since 1992. Any case suspicious of contamination is investigated.

The chest X-rays were described by physicians who had not been blinded to the fact that the patient had participated in the project. This could lead to under-reporting of results suggestive of TB, but more likely to over-reporting, since the sputum culture results were known to the physician. However, chest $\mathrm{X}$-ray is not always suggestive of TB in patients with pulmonary $\mathrm{TB}^{21}$ and chest X-ray may certainly be normal at an early disease stage.

Only $7(19.4 \%)$ of the 36 TB cases were smear-positive, implying that smear microscopy is not sufficient to identify TB cases in screening programmes. Smear microscopy status is an indicator of infectiousness, ${ }^{22}$ although transmission from smearnegative $\mathrm{TB}$ cases can be seen. ${ }^{23}$ At treatment initiation, $24(70.6 \%)$ of $34 \mathrm{~TB}$ cases tested in our study were smearnegative. In comparison $44.4 \%$ of all pulmonary TB cases in Denmark in 2013 were smear-negative. ${ }^{24}$ Although only 12 provided two sputum samples or more, this indicates that they were found at an early, less infectious stage. This is important, because it makes a favourable treatment outcome for the patient more likely, and to reduce transmission and gain disease control.

In our study median treatment delay (from screening date to treatment initiation) was 32 days and median locating delay (from screening result to treatment initiation) was 8 days. Meanwhile disease burden and infectivity may increase and especially homeless participants may become increasingly difficult to relocate. NAATs provide results faster (less than $2 \mathrm{~h}$ ) than culture and have a higher sensitivity than smear microscopy. ${ }^{18}$ We used the commercially available BD ProbeTec, a system with a similar overall performance when compared with the Xpert MTB/RIF system (Cepheid). ${ }^{25}$ The sensitivity of NAAT in our screening study was surprisingly low (40.0\%). Patients found by screening will probably be found at an earlier stage of their TB disease. Thus, a large part of our TB cases were smear-negative and NAAT-negative.

We found a very high TB prevalence of 2233/100 000 in the study population. Although no symptom screening was done, persons with no symptoms will not be able to produce a sputum sample and will be less likely to participate in the screening programme. Therefore, our study population probably represents a subgroup of socially marginalised persons in Copenhagen with a higher prevalence of TB than the rest of this population. Most TB cases were found the first time the participant was screened, but an additional $12 \mathrm{~TB}$ cases were found when the participants were screened repeatedly over time. Although repeated testing in itself results in a higher sensitivity, ${ }^{26}$ this suggests ongoing transmission in the screened population. Furthermore, all genotyped TB cases identified were clustered and $68.6 \%$ were part of the biggest Danish cluster called C2/1112-15, which also suggests a lack of disease control in these high-risk groups as previously documented. ${ }^{14-16}$ 
One limitation to our study is that the only available baseline characteristics of the screened population are age and sex. This limits the possibility to generalise the results to other settings. Furthermore $72.2 \%$ of the TB cases identified were Greenlandic Inuit. We do not know the distribution of the ethnicity of the screened population, so we do not know whether this reflects the ethnicity of the screened population or a specifically high TB incidence among Greenlandic Inuit in Copenhagen. However, although no data are available, it is the impression of the screening team that primarily Danes, Greenlandic Inuit and Somalis were screened, so both explanations are probably true.

In low-incidence countries, TB is concentrated in high-risk groups and screening of these groups has been recommended by WHO. ${ }^{5} 2728$ Chest X-ray has obvious advantages such as immediate availability, high sensibility and the fact that patients can be screened independently of symptoms. If a mobile X-ray unit is available it is possible to perform community based screening, which is pivotal to ensure participation among socially marginalised persons. Our results suggest that sputum culture is a promising, maybe even better alternative to mobile X-ray screening. Although results are not available instantly, sputum culture can be repeated with small intervals (no radiation) and has a higher sensitivity; indeed $22.2 \%$ of the cases identified in our study would not have been identified by chest X-ray. Furthermore our results suggest that the $\mathrm{TB}$ cases were identified at an early, less infectious stage of disease. This is important, because an early diagnosis is essential in order to reduce transmission and help gain disease control in high-risk groups. NAATs may provide results faster and could be added to culture using the same sputum sample, but the evidence from our study was not promising. Further studies are needed to confirm our findings and to clarify if similar results can be obtained in other settings.

Contributors SGJ, NWO, NS, TL, TW and AK-J designed the study and the database. SGJ and NWO obtained data from the medical records at Gentofte Hospital and SGJ, TL and MKP retrieved information from Statens Serum Institute regarding results of sputum samples. All authors contributed to data analysis and interpretation and writing of the manuscript. All authors had full access to all data in the study and can take responsibility of the integrity of the data and the accuracy of the data analysis. NS is guarantor. All authors have seen and approved the final version of the manuscript.

Funding The Capital Region of Denmark and the Danish Lung Association.

Competing interests None declared.

Ethics approval The study was a retrospective register study as confirmed by the Central Ethics Committee of Denmark (protocol no. H-1-2013-FSP-64).

Provenance and peer review Not commissioned; externally peer reviewed.

Data sharing statement Relevant anonymised patient level data will be available from the authors on request, when all papers related to the data have been published.

\section{REFERENCES}

1 Beijer U, Wolf A, Fazel S. Prevalence of tuberculosis, hepatitis C virus, and HIV in homeless people: a systematic review and meta-analysis. Lancet Infect Dis 2012;12:859-70.

2 Fazel S, Geddes JR, Kushel M. The health of homeless people in high-income countries: descriptive epidemiology, health consequences, and clinical and policy recommendations. Lancet 2014;384:1529-40.

3 Zenner D, Southern J, van Hest R, et al. Active case finding for tuberculosis among high-risk groups in low-incidence countries. Int J Tuberc Lung Dis 2013;17:573-82.

4 Story A, Murad S, Roberts W, et al. Tuberculosis in London: the importance of homelessness, problem drug use and prison. Thorax 2007;62:667-71.
5 World Health Organization. Framework towards tuberculosis elimination in low-incidence countries. WHO, 2014. http://who.int/tb/publications/elimination_ framework/en/ (accessed Mar 2014).

6 Jimenez-Fuentes MA, Auge CM, Gomez MN, et al. Screening for active tuberculosis in high-risk groups. Int J Tuberc Lung Dis 2014;18:1459-65.

7 Paquette K, Cheng MP, Kadatz MJ, et al. Chest radiography for active tuberculosis case finding in the homeless: a systematic review and meta-analysis. Int I Tuberc Lung Dis 2014;18:1231-6.

8 de Vrief G, van Hest RA, Richardus JH. Impact of mobile radiographic screening on tuberculosis among drug users and homeless persons. Am J Respir Crit Care Med 2007;176:201-7.

9 Bernard C, Sougakoff W, Fournier A, et al. Impact of a 14-year screening programme on tuberculosis transmission among the homeless in Paris. Int I Tuberc Lung Dis 2012;16:649-55.

10 Story A, Aldridge RW, Abubakar I, et al. Active case finding for pulmonary tuberculosis using mobile digital chest radiography: an observational study. Int J Tuberc Lung Dis 2012;16:1461-7.

11 Kimerling ME, Shakes CF, Carlisle R, et al. Spot sputum screening: evaluation of an intervention in two homeless shelters. Int J Tuberc Lung Dis 1999;3:613-19.

12 Badiaga S, Richet $\mathrm{H}$, Azas $\mathrm{P}$, et al. Contribution of a shelter-based survey for screening respiratory diseases in the homeless. Eur J Public Health 2009;19:157-60

13 Svensson E, Suppli CH, Andersen PH. EPI-NEWS. National surveillance of communicalble diseases, tuberculosis, Copenhagen, Denmark. Statens Serum Institut, 2014. http://www.ssi.dk/Aktuelt/Nyhedsbreve/EPI-NYT/2014/Uge $\% 204 \%$ 20-\%202014.aspx (accessed Mar 2014).

14 Kamper-Jorgensen Z, Andersen AB, Kok-Jensen A, et al. Clustered tuberculosis in a low-burden country: nationwide genotyping through 15 years. J Clin Microbiol 2012;50:2660-7

15 Lillebaek T, Andersen AB, Seersholm NJ, et al. [Continued problems with tuberculosis among Danes and Greenlanders in Denmark and the need for reinforced control—a systematic review]. Ugeskr Laeger 2012;174: 2696-701.

16 Lillebaek T, Andersen AB, Rasmussen EM, et al. Mycobacterium tuberculosis outbreak strain of Danish origin spreading at worrying rates among Greenland-born persons in Denmark and Greenland. J Clin Microbiol 2013;51:4040-4.

17 Rådet for Socialt Udsatte. I Grønland er jeg for dansk, og i Danmark er jeg 'bare' Grønlænder. Denmark: Rådet for Socialt Udsatte, 2014. http://www.udsatte.dk/dyn/ resources/Publication/file/8/58/1390811813/

i-groenland-er-jeg-for-dansk-og-i-groenland-er-jeg-bare-groenlaender-online-version. pdf (accessed Mar 2014).

18 Drobniewski F, Nikolayevskyy V, Balabanova Y, et al. Diagnosis of tuberculosis and drug resistance: what can new tools bring us? Int J Tuberc Lung Dis 2012;16:860-70.

19 Solsona J, Cayla JA, Nadal J, et al. Screening for tuberculosis upon admission to shelters and free-meal services. Eur J Epidemiol 2001;17:123-8.

20 Barry MA, Wall C, Shirley L, et al. Tuberculosis screening in Boston's homeless shelters. Public Health Rep 1986;101:487-94.

21 Wilcke JT, Askgaard DS, Nybo JB, et al. Radiographic spectrum of adult pulmonary tuberculosis in a developed country. Respir Med 1998;92:493-7.

22 Lohmann EM, Koster BF, le Cessie S, et al. Grading of a positive sputum smear and the risk of Mycobacterium tuberculosis transmission. Int J Tuberc Lung Dis 2012;16:1477-84.

23 Tostmann A, Kik SV, Kalisvaart NA, et al. Tuberculosis transmission by patients with smear-negative pulmonary tuberculosis in a large cohort in the Netherlands. Clin Infect Dis 2008;47:1135-42.

24 Voss S, Andersen PH, Svensson E. EPI-NEWS uge 3-2015. National surveillance of communicalble diseases, tuberculosis, Copenhagen, Denmark. Statens Serum Institut, 2015. http://www.ssi.dk/Aktuelt/Nyhedsbreve/EPI-NYT/2015/Uge\%203\% 20-\%202015.aspx (accessed March 2014).

25 Antonenka U, Hofmann-Thiel S, Turaev L, et al. Comparison of Xpert MTB/RIF with ProbeTec ET DTB and COBAS TaqMan MTB for direct detection of M. tuberculosis complex in respiratory specimens. BMC Infect Dis 2013;13:280.

26 Mase SR, Ramsay A, Ng V, et al. Yield of serial sputum specimen examinations in the diagnosis of pulmonary tuberculosis: a systematic review. Int J Tuberc Lung Dis 2007;11:485-95.

27 World Health Organization. Systematic screening for active tuberculosis principles and recommendations. WHO, 2013. http://who.int/tb/tbscreening/en/) (accessed Mar 2014).

28 Lonnroth K, Corbett E, Golub J, et al. Systematic screening for active tuberculosis: rationale, definitions and key considerations. Int J Tuberc Lung Dis 2013;17:289-98. 